International Journal of Engineering \& Technology, $7(2.21)(2018) 291-295$
International Journal of Engineering \& Technology
WPC
Website: www.sciencepubco.com/index.php/IJET
Research paper

\title{
Mining of high dimensional data using enhanced clustering approach
}

\author{
S. Sivakumar ${ }^{*}$, Kumar Narayanan², Swaraj Paul Chinnaraju ${ }^{3}$, Senthil Kumar Janahan ${ }^{4}$ \\ ${ }^{I}$ Department of Computer Science \& Engineering, Vels Institute of Science, Technology \& Advanced Studies(VISTAS), Chennai, India. \\ ${ }^{2}$ Department of Computer Science \& Engineering, Vels Institute of Science, Technology \& Advanced Studies(VISTAS), Chennai, India. \\ ${ }^{3}$ Department of Computer Science \& Engineering, Vels Institute of Science, Technology \& Advanced Studies(VISTAS), Chennai, India. \\ ${ }^{4}$ Department of Computer Science \& Engineering, Vels Institute of Science, Technology \& Advanced Studies(VISTAS), Chennai, India. \\ *Corresponding author E-mail:ssivacse@gmail.com
}

\begin{abstract}
Extraction of useful data from a set is known as Data mining. Clustering has top information mining process it supposed to help an individual, divide and recognize numerous data from records inside group consistent with positive similarity measure. Clustering excessive dimensional data has been a chief undertaking. Maximum present clustering algorithms have been inefficient if desired similarity is computed among statistics factors inside the complete dimensional space. Varieties of projected clustering algorithms were counseled for addressing those problems. However many of them face problems whilst clusters conceal in some space with low dimensionality. These worrying situations inspire our system to endorse a look at partitional distance primarily based projected clustering set of rules. The aimed paintings is successfully deliberate for projects clusters in excessive huge dimension space via adapting the stepped forward method in $\mathrm{k}$ Mediods set of pointers. The main goal for second one gadget is to take away outliers, at the same time as the $1 / 3$ method will find clusters in numerous spaces. The (clustering) technique is based on the adequate Mediods set of guidelines, an excess distance managed to set of attributes everywhere values are dense.
\end{abstract}

\section{Introduction}

\section{Data mining-introduction}

Huge value of information is generated every day. It's miles very difficult to discover beneficial facts among them. Extracting or "mining" information gained from enormous quantity of records is known as Data Mining. These tools are used to discover the secret facts, useful styles from the information. Information mining is as famous as "know-how Discovery in Databases" (KDD). Statistics Mining is a multi-disciplinary subject which includes device getting to know, Measurements, Visualization, Database, professional systems.

The method of analyzing information from diff views and summarizing it into beneficial facts with a purpose to increase profits, decision making abilities and to decrease price is referred to as facts mining. It analyses statistics in special dimensions and identifies the connection among facts. Records can be classified as follows:

- Operational Data - Sales, Purchase, accounting and inventory

- Non Operational Data - Forecast data

- Meta Data - Data about data

- Information and Knowledge

Statistics can be drawn with the help of institutions, connections and styles amongst records. Extraction of expertise can be obtained from the hidden facts with the aid of the usage of records mining tools and techniques. Records mining assistances groups to classify relationship amongst internal elements which include team of workers skills, inventory requirements and additionally to categorise outside elements such as marketplace competition, monetary indicators.
Statistics mining algorithms face many demanding conditions like scalability, imparting the extracted information in a easy way to customers, using suitable technique to offer the information in a widespread manner and so on. Statistics Mining has many realistic packages. Large banks and coverage companies use KDD to analyze their consumer files. The vital information mining strategies are

- Data mining-it's miles a studies approach this is capable of understand exciting relationships amongst statistics objects in the facts set. marketplace basket assessment is the high-quality example for affiliation rule mining (Agrawal et al 1993). It basically analyses patron purchasing for conduct and produces association amongst records devices presented. It allows shops to plot their market techniques. affiliation rules are determined through using association assessment. association recommendations display characteristic-rate conditions that rise up regularly in a given set of statistics. association tips are of the form X. Y way that tuples which fulfill the circumstance in $X$ also are likely to satisfy the situation in $\mathrm{Y}$ inside the database.

- Category - it's also referred to as supervised learning. It has steps. Within the first step a model is built to provide an explanation for pre-defined set of statistics lessons or standards. Some attributes are referred to as elegance label attributes as they decide pre-defined training. Institution of records tuples, that are used to build the version is referred to as training facts set and character tuples are called education samples. Within the second step, same version is used for class.

- Clustering - A cluster is described as a set of records objects which are much like each other and consequently can be dealt with together as one group. 
Clustering is called unsupervised learning as there aren't any pre-described instructions. The class labels of every training pattern as well as the amount of lessons aren't appeared earlier. it is a manner of grouping comparable items together. The outstanding of a clustering method is calculated by way of its functionality to discover all the hidden styles.

- Outlier evaluation - some facts devices in a database do now not have a look at other devices or do now not follow famous conduct of the records. Those gadgets are referred to as outliers (Aggarwal and $\mathrm{Yu}$ 2001). maximum of the records mining algorithms are capable of discover outliers and dispose of them as noise or exceptions.

Every statistics mining technique works on awesome kinds of information, uses specific running ideas, produces first rate output.

\section{Scope}

Today theoretical effects show that data elements in a set have a propensity to be greater in addition spaced due to the fact the dimension of the gap will boom, the parts of the statistics detail are independently and similarly dispensed. Despite the fact that the circumstance is not often fulfilled in actual packages, it however will become a lot much less significant to distinguish data factors based totally on a distance or a identically diploma computed using all the dimensions. Those consequences offer an motive in the back of the awful typical overall work done of traditional distance primarily based definitely clustering set of rules on such facts gadgets.

\section{Aim}

A number of projected clustering algorithms were recommended but, maximum of them run into difficulties it hides subspaces with very low dimensions. Those worrying conditions inspire our efficient work done suggest a partitional distance primarily based absolutely executed clustering set of guidelines.

\section{Clustering}

Clustering is a commonplace statistics mining approach that is preferred-for to assist the customer to discover and understand the structure or grouping of the data in the set in step with a certain resemblance degree. At the same time as doing cluster evaluation, we first partition the set of information into groups based mostly on information similarity after which assign the labels to the companies. Clustering techniques can be categorized into the subsequent classes.

\section{Partitioning approach}

Count on we are given a database of ' $n$ ' devices and the partitioning method constructs ' $\mathrm{k}$ ' partition of facts. each partition will constitute a cluster and ok $=n$. It technique that it'll classify the statistics into ok agencies, which fulfill the subsequent necessities. every corporation consists of at least one item.

\section{Hierarchical techniques}

This approach creates a hierarchical decomposition of the given set of information devices. We are able to classify hierarchical strategies on the concept of the manner the hierarchical decomposition is commonplace. There are techniques proper right here. Agglomerative method and Divisive technique.

\section{Agglomerative method}

This method is also diagnosed as the lowest-up method. In this, we begin with each object forming a separate institution. It maintains on merging the devices or corporations which is probably near every other. It continues on doing so until all the businesses are merged into one or till the termination situation holds.

\section{Divisive method}

This approach is likewise known as the pinnacle-down method. On this, we begin with all of the objects inside the same cluster. within the non-prevent generation, a cluster is split up into smaller clusters. It is down till every item in a single cluster or the termination scenario holds. This technique is inflexible, i.e., as quickly as a merging or splitting is finished, it may in no manner be undone.

\section{Density-based completely technique}

This approach is based totally mostly on the perception of density. The easy idea is to keep growing the given cluster as long as the density inside the neighborhood exceeds a few threshold, i.e., for every information point inner a given cluster, the radius of a given cluster has to include as a minimum a minimal big variety of factors.

\section{Grid-based totally technique}

In this, the devices together shape a grid. The object area is quantized into finite quantity of cells that shape a grid shape. The number one advantage of this approach is fast processing time. It's far reliant on simplest at the style of cells in every duration within the quantized place.

\section{Version-based totally techniques}

On this method, a version is hypothesized for each cluster to locate the good in shape of data for a given model. This method finds the clusters thru clustering the density characteristic. It mirrors spatial distribution of the statistics factors. This method moreover presents a manner to routinely decide the style of clusters based mostly on great records, taking outlier or noise into account. It therefore yields strong clustering techniques.

\section{Constraint-based approach}

Here the clustering is done with the useful resource of the incorporation of client or software program-oriented constraints. A problem refers to the client expectation or the houses of desired clustering results. Boundaries provide us with an interactive way of communication with the clustering manner. Constraints may be unique with the resource of the client or the software requirement.

\section{Relevant Works}

\section{List of modules}

- Attribute relevance analysis

- Sparseness Estimation

- Outlier handling

- Discovery of projected clusters

\section{Attribute relevance analysis}

The purpose is to find out all proportions in displayed dataset a few cluster on by manner of identifying having low dense places and they will be positioned in every size. 
The identified thing constitute vary with suitable proportions of clusters.

\section{Sparseness Estimation}

- By the help characteristic significance evaluation, the sparseness level yij are identified for numerous proportions.

- The smallest assessment of yij shows stable place and most evaluation indicates thin region. Likewise, several yij values predicted for numerous spatial pics for various shapes.

- Photos having higher standards of yij specify some skinny regions.

- The feature with fewer values of yij suggest the opaque areas.

\section{Outlier dealing with}

- The final output number one segment, the purpose is to apprehend and dispose of points dataset.

\section{Existing System}

- In clustering high dimensionality affords a dual trouble.

- First, the life of beside the point attributes gets rid of any wish on clustering tendency.

- The second one trouble is the dimensionality curse that may be a lack of statistics separation in high dimensional space.

- Many spatial clustering algorithms cling on indices in spatial datasets to facilitate quick seek of the closest pals.

- A number of projected clustering algorithms configures the drawback in figuring out very low dimensional executed clusters combined in excessive spacial area.

- A few partitioned projected clustering set of rules uses identical characteristics it includes each and every dimension with the intention of locating an starting point of cluster

- The usage of some heuristics are determined by the every dimensions of cluster.

- A few existing projected clustering set of rules needs a person to offer the common space dimensionality.

\section{Limitations}

- Iteratively computes a good medoid for every cluster.

- A similarity feature that uses all dimensions misleads the applicable dimensions detection mechanism and adversely affects the performance of the algorithms.

- The locality is formed by the method of primarily depends on the whole space dimensionality

- Always not useful control the drawbacks in datasets having low priority dimension executed cluster.

- The user parameters of some set of rules are difficult to decide an incorrect desire by means of the person having greater accuracy.

\section{Advantages}

- The final results of every segment acts as an enter for the subsequent section.

- The very last final results would be the projected clusters of excessive dimensional subspaces.

- Expected clusters have got to be dense

- Dense areas are involved in the space calculation.

\section{Experimental setup and results}

\section{Experimental setup}

The implementation of algorithm was carried out in asp.net framework using C\# language and back end as MSSQL server 2008. ASP.Net C\# is a simple, modern and object-oriented programming language developed by Microsoft. This language is based on $\mathrm{C}$ and $\mathrm{C}++$ programming language. It is a structural language and also produces efficient programs. This $\mathrm{C \#}$ language is compiled on a various types of computer platforms. It is a component oriented language and also easy to understand and learn. C\# language is a part of .NET framework.

\section{Experimental results}

GUI for Mining Projected group of objects in Dimensional spaces

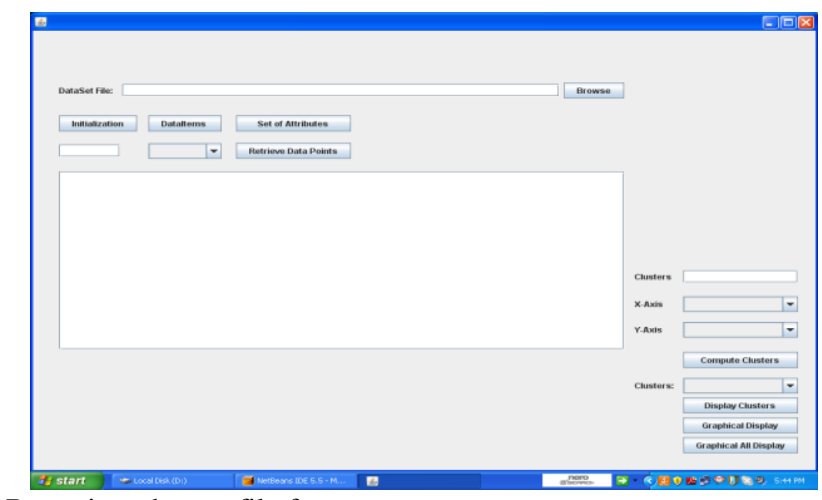

Browsing dataset file from system

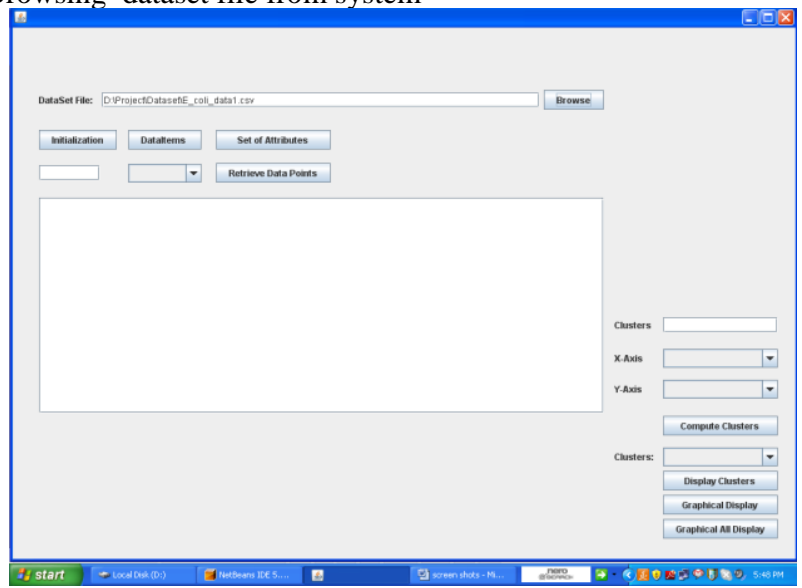

\section{Result analysis}

The most illustrative algorithm $\mathrm{K}$ Medoids, $\mathrm{K}$ Means and suggested algorithm were analyzed based on their basic approach for large data set

Table 1: Number of clusters and execution time (in milliseconds)

\begin{tabular}{|c|c|c|}
\hline $\begin{array}{c}\text { Number of } \\
\text { Clusters }\end{array}$ & $\begin{array}{c}\text { Execution } \\
\text { Time } \\
\text { K-Mean } \\
\text { Algorithm }\end{array}$ & $\begin{array}{c}\text { Execution Time } \\
\text { K-Mediod Algorithm }\end{array}$ \\
\hline 2 & 21348 & 20325 \\
\hline 3 & 42170 & 40380 \\
\hline 4 & 68510 & 67210 \\
\hline 5 & 74360 & 73340 \\
\hline
\end{tabular}




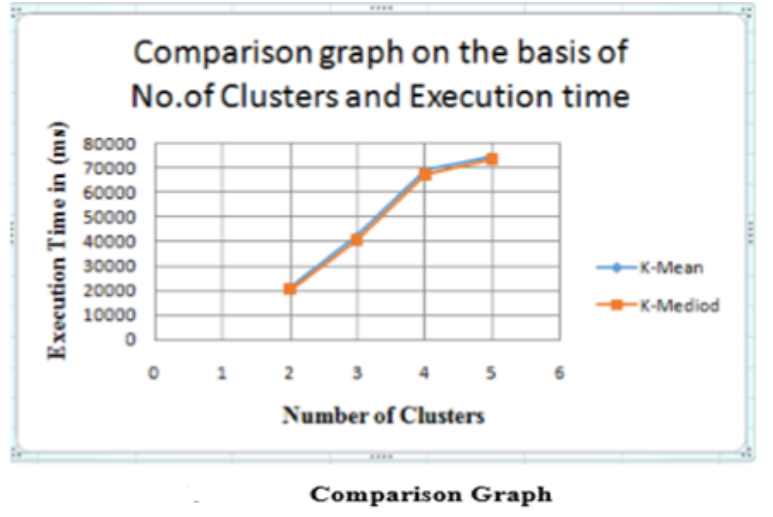

It is cleared from the table 1 and relevant graph figure 4 that irrespective of number of clusters the execution time occupied by $\mathrm{K}$ Medoid algorithm is usually less than that of K-Mean algorithm.

Table-2: Number of records and execution time (In milliseconds)

\begin{tabular}{|c|c|c|}
\hline $\begin{array}{c}\text { Number of } \\
\text { Records }\end{array}$ & $\begin{array}{c}\text { Execution } \\
\text { Time } \\
\text { K-Mean } \\
\text { Algorithm }\end{array}$ & $\begin{array}{c}\text { Execution Time } \\
\text { K-Mediod Algorithm }\end{array}$ \\
\hline 300 & 94242 & 82232 \\
\hline 400 & 112371 & 110301 \\
\hline 500 & 138523 & 129202 \\
\hline 600 & 164362 & 139561 \\
\hline
\end{tabular}

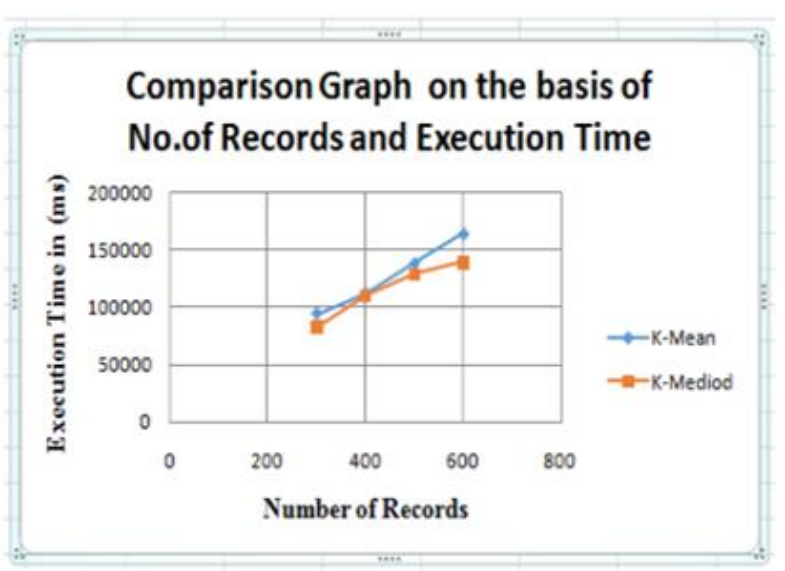

Comparison Graph on the basis of Number of records and execution time

Above table and figure shows the comparison between Kmean and K-Medoid Algorithms. As the graph shows that irrespective of amount of records and the execution time occupied by K Medoid algorithm is usually less than that of KMean algorithm. At the most amount of records is increased than the execution time occupied by K-Medoids is less than the K Means Algorithm.
Table 3: Number of clusters and execution time (in milliseconds)

\begin{tabular}{|c|c|c|}
\hline $\begin{array}{c}\text { Number of } \\
\text { Clusters }\end{array}$ & $\begin{array}{c}\text { Execution } \\
\text { Time } \\
\text { K-Mediod } \\
\text { Algorithm }\end{array}$ & $\begin{array}{c}\text { Execution Time } \\
\text { Extended K-Mediod } \\
\text { Algorithm }\end{array}$ \\
\hline 2 & 20325 & 19311 \\
\hline 3 & 40380 & 38595 \\
\hline 4 & 67210 & 65916 \\
\hline 5 & 73340 & 72321 \\
\hline
\end{tabular}

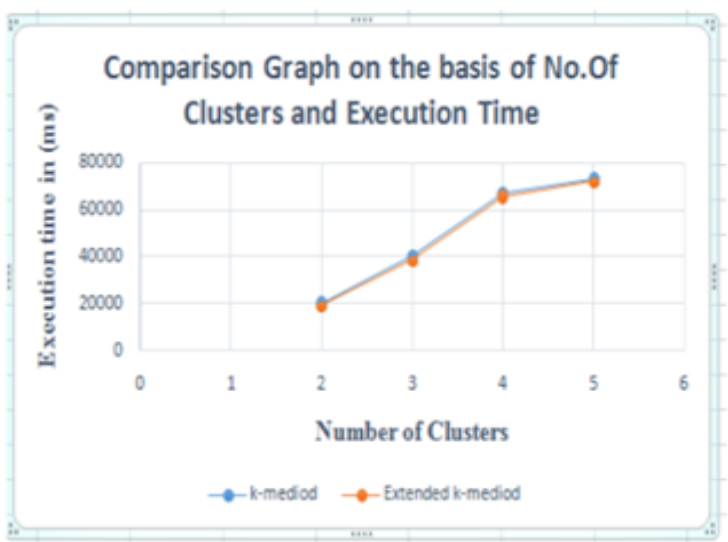

Comparison graph on the basis of number of clusters and execution time

It is cleared from the table 3 and relevant figure 6 that irrespective of number of clusters the execution time occupied by Extended $\mathrm{K}$ Medoid algorithm is usually less than that of K Medoid algorithm.

Table 4.Number of records and execution time (In
milliseconds)
\begin{tabular}{|c|c|c|}
\hline $\begin{array}{c}\text { Number of } \\
\text { Records }\end{array}$ & $\begin{array}{c}\text { Execution } \\
\text { Time } \\
\text { K-Mediod } \\
\text { Algorithm }\end{array}$ & $\begin{array}{c}\text { Execution Time } \\
\text { Extended K-Mediod } \\
\text { Algorithm }\end{array}$ \\
\hline 300 & 82232 & 70222 \\
\hline 400 & 110301 & 108231 \\
\hline 500 & 129202 & 119881 \\
\hline 600 & 139561 & 114760 \\
\hline
\end{tabular}

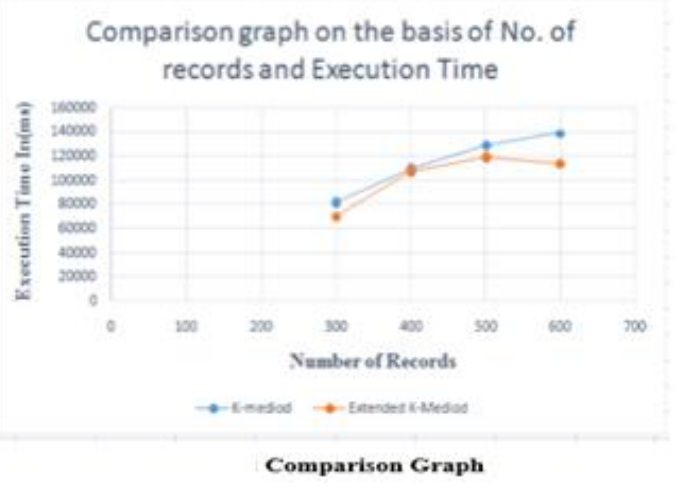

Above table and figure shows the comparison between KMedoid Algorithm and Modified K-Medoids Algorithms. As the graph shows that irrespective of number of records the execution time occupied by Extended K Medoid algorithm is usually less than that of K Medoid algorithm. The extended K Medoid performs better than K Medoid algorithm in most of the cases.

\section{Conclusion}

We have suggested unaltered Medoid algorithmic rule for enhancing perfectly and scalability for the research of huge datasets. The result from number of clusters and records shows that the $\mathrm{K}$ Medoid Algorithmic rule has good performance in terms of duration time, quality of clusters. No. of grouped objects 
and history of information than K-Means\&K-Medoid Algorithmic rules. Extended $\mathrm{K}$ Medoid Algorithmic rule is corrected using sample real employee datasets and results are related with $\mathrm{K}$ Means \&K-Medoid Algorithmic rules. In the future work, comparison is made of the extended KMedoids Algorithm with other algorithms in order to substantiate and fetch improvement in the study. They can use the different methods to further enhance the efficiency and scalability by decreasing the completing time.

\section{References}

[1] Agrawal R, Gehrke J, Gunopulos D \& Raghavan P, "Automatic Subspace Clustering of High Dimensional Data", Data Mining and Knowledge Discovery, Vol.11, No.1, (2005), pp.5-33.

[2] Liu H \& Yu L, "Toward Integrating Feature Selection Algorithms for Classification and Clustering", IEEE Trans. Knowledge and Data Eng., Vol.17, No.4, (2005), pp.491-502.

[3] Yip KYL, Cheng DW \& Ng MK, "On Discovery of Extremely Low-Dimensional Clusters Using Semi-Supervised Projected Clustering", Proc. 21st Int'l Conf. Data Eng, (2005), pp. 329-340.

[4] Lung $M$ \& Mamoulis N, "Iterative Projected Clustering by Subspace Mining", IEEE Trans. Knowledge and Data Eng., Vol.17, No.2, (2005), pp.176-189.

[5] Bouguessa M, Wang S \& Jiang Q, “A K-Means-Based Algorithm for Projective Clustering”, Proc. 18th IEEE Int'l Conf. Pattern Recognition, (2006), pp.888-891.

[6] Bouguessa M, Wang S \& Sun H, "An Aim Approach to Cluster Validation”, Pattern Recognition Letters, Vol.27, No.13, (2006), pp.1419-1430.

[7] Angiulli F \& Pizzuti C, "Outlier Mining in Large HighDimensional Data Sets", IEEE Trans. Knowledge and Data Eng., Vol.17, No.2, (2005), pp.369-383.

[8] Li T, "A Unified View on Clustering Binary Data", Machine Learning, Vol.62, No.3, (2006), pp.199-215.

[9] Patrikainen A \& Meila M, "Comparing Subspace Clusterings", IEEE Trans. Knowledge and Data Eng., Vol.18, No.7, (2006), pp.902-916.

[10] Tjaden B, "An Approach for Clustering Gene Expression Data with Error Information”, BMC Bioinformatics, Vol.7, No.17, (2006).

[11] Doherty KAJ, Adams RG \& Davey N, "Unsupervised Learning with Normalized Data and Non-Euclidean Norms", Applied Soft Computing, Vol.7, No.17, (2007), pp.203-210. 